\title{
The effect of content and model type in sidebar advertising on middle-aged women's online purchases of anti-aging products
}

\begin{abstract}
With marketing strategies, for anti-aging products targeted to middle-aged women, changing from traditional mass media to sidebar advertising on social media sites like Facebook, it is important to understand if the middle-age women purchased these anti-aging products based on the content and model type portrayed in the sidebar advertisement. Previous studies about advertising to middle-aged women indicated advertisements did not make a connection with women. This study examined whether or not sidebar advertisements created with credibility, informativeness, and believability would make a connection with middle-aged women motivating them to buy an antiaging product. Three-hundred and eighty-five women participated in the testimonial study. Results indicated that there was significant difference found in middle-aged women's perception of the believability to purchase from an advertisement depicted in an online sidebar advertisement for an anti aging product.
\end{abstract}

Keywords: middle-aged women, facebook, anti-aging, sidebar advertising, social media, gratification theory
Volume 4 Issue I - 2018

\author{
Jenifer Roberts \\ Merchandising and Fashion Design, Missouri State University, \\ USA
}

Correspondence: Jenifer Roberts, Merchandising and Fashion Design, Missouri State University, 90 I S. National Avenue, Springfield, MO 65897, USA, Tel 4I72343629, Fax 4I7836434I, Email Jeniferroberts@missouristate.edu

Received: December 06, 2017 | Published: January 09, 2018

\section{Introduction}

In the United States, the beauty industry reached $\$ 62.46$ billion in sales in 2016 ("Revenue," 2015). A large portion of the beauty industry sales is anti-aging beauty products ("Anti-aging Products," 2009). There are approximately 48 million middle-aged women between 30 and 55years of age (Census.Gov, 2011). The most used social media site for middle-aged women is Facebook, which is currently the largest social media outlet for advertising world-wide, averaging four to six advertisements per site ("Social Media," 2014). Beauty product marketers have not optimally targeted middle-aged women through sidebar advertisements on social media sites. ${ }^{1}$ Sidebar advertisement is an internet advertisement in the form of a vertical or horizontal bar on a website that contains graphics, photographs, or text to promote a product presented on social media websites. ${ }^{2,3}$

Middle-aged women between the ages of 30 and 55 are an ideal, underutilized market for the advertisement of beauty enhancement anti-aging products. ${ }^{4}$ Middle-aged women frequently desire to look younger than their chronological age. ${ }^{5,6}$ With a captive audience of over 56 million middle-aged Facebook users, a majority of middleaged women will acquire information regarding anti-aging products from Facebook via their peers ("Social Media," 2014). Millions of middle-age women are exposed to sidebar advertisements featuring anti-aging products for the billion-dollar personal beauty product industry. ${ }^{7}$ Williams \& Page $^{8}$ contend that unlike younger women who are drawn more to the visual aspects of products, middle-aged women are more likely to prefer reading labels and product reviews carefully when making buying decisions. Internet beauty product advertisements generally depict models that are young, age-inappropriate and do not include information that would contribute to middle-aged women's purchase decision, ${ }^{9-12}$ although research suggests that these factors are what is desired. ${ }^{8,12}$

According to uses and gratification theory (UGT), people are more likely to be drawn to products that depict models who are similar to the purchaser. ${ }^{13-15}$ Middle-aged women are more likely to purchase products based on advertisements that depict age reflective models. Consumers find advertising more credible, believable, and informative when a product advertisement is personalized toward the perceptions of the customer. ${ }^{16,17}$ Internet sidebar advertisements that include ageappropriate models and credible information (e.g., testimonials and statistics) may better target middle-aged women for buying beauty enhancement anti-aging products. ${ }^{9}$

Middle-aged women's expectations in what may influence them regarding sidebar advertisements for anti-aging beauty products, specifically on Facebook, has not been wholly represented or salient in the research literature. Much of what is known about marketing to middle-aged women is based on research regarding traditional mass media advertising in print, TV, and radio. ${ }^{18}$ This research focused on body image, ${ }^{19,20}$ type of model,${ }^{18}$ advertisement placement, ${ }^{21}$ and psychological effects ${ }^{22}$ of how advertisements affect women. Although traditional media continues to be of importance, research on social media advertising is of importance as well in that it is a large part of the advertising mix with approximately $38 \%$ on banner advertisement and $29 \%$ on paid search advertising. ${ }^{23}$ The effects of content and model types in relation to credibility, believability, and informativeness of sidebar advertisements is of interest in that social media affects sales. ${ }^{23}$

As online advertising continues to grow, averaging $\$ 76.8$ million a year in advertisement dollars ("Revenue," 2015), research is warranted to understand middle-aged women and what type of online advertising prompts purchase. Currently, middle-aged women are the fastest growing market segment in America, comprising $28 \%$ of online purchases ("Age Matters," 2014). For beauty companies to maintain their market shares and penetrate their business, it is important for marketers to understand what motivates this consumer to buy online. 


\section{Traditional to online advertisement}

The relationship between women's perceptions and the presentation of beauty products in traditional mass media has been well documented. ${ }^{7,20-22}$ Marketers for anti-aging beauty enhancement products are moving away from traditional mass media to online venues such as Facebook. Online advertisements are more cost effective than traditional mass media such as television. Currently, the cost to reach 1,000 people through an advertisement on Facebook is $\$ 0.25$, which is only $1 \%$ of the cost of a television advertisement, ${ }^{24}$ whereas print advertisements placed in People magazine average $\$ 360,000$ for a full page advertisement, which costs $\$ 20$ or more per 1000 people, that reaches approximately 44.2 million people (People, 2015). Internet sidebars for anti-aging beauty products are still new. ${ }^{24}$ There is only a superficial understanding of the factors that create effective sidebar advertisements for middle-aged women. Sidebar advertisements include additional content not available in traditional advertisements. For example, anti-aging beauty sidebars can be tailored toward a specific target customer for a more personal advertisement. Social media advertisements offer the opportunity to include testimonials, consumer opinions, and statistical information that is based on the consumers' online profile or tracking.

For decades, researchers have found that stereotyping, sexism, and ageism are problems in traditional advertisement content. Given that more than 53.1 million U.S. middle-aged women are seeking information online about anti-aging appearance enhancement products ("2011 Social Media Consumer Trend and Benchmark Report," 2011), it is important for marketers in the multi-billion-dollar antiaging product industry to understand clearly what motivates middleaged women to purchase these anti-aging beauty products. Based on past research there is a problem with the content in traditional advertisements when it comes to middle-aged women. As marketers continue to use the internet it is important to create advertisements with credibility, informativeness, believability that connects with middleaged women. Marketers have, in traditional media, paid less attention to model types and content than seen in social media advertisements. ${ }^{9}$ Positive social media advertisements can benefit a beauty company by increasing the consumer base and sales. The current research seeks to provide insight in how the effects of content and model type in sidebar advertising for anti-aging beauty products helps to motivate or not motivate these consumers to purchase the product.

Theoretical Framework: The theoretical underpinning of this research is the Uses and Gratification Theory (UGT). UGT has been used for decades, since first proposed by Herzog ${ }^{25,26}$ as a sociological explanation for why consumers expressed a single mass media preference to help them satisfy a specific need. Close to a decade later, Schramm (1961), continued research about UGT and developed a formula called fraction of selection, which mathematically calculated how often users sought to use media based on a reward, effort, difficulty, and frequency of seeing an advertisement. ${ }^{13}$ Years later in 1973, Katz et al. ${ }^{14}$ introduced five elements: The audience element for Facebook includes active participants such as middle-aged women who use this social media site as more of a pastime than a goal-driven activity Katz et al. ${ }^{14}$ The media competition element is a combination of mass communication and media fulfillment of human need satisfaction, whereas need can be met through mass media consumption Katz et al. ${ }^{14}$ The self-awareness element allows for individuals to understand their interests and motives when presented with comprehensible and familiar verbal formulation. The self-awareness element that Katz et al. ${ }^{14}$ describes with respect to UGT is that people want to be able to share their interest and motives in certain situations. The judgement element focuses on certain affinities and indifferences Katz et al. ${ }^{14}$ Meaning, the relatability a consumer has to the informational content or spokesperson, as well as the irrelevance or lack of connection to the spokesperson in the advertisement. The difference between Facebook advertising and traditional mass media advertising is that Facebook advertising can target specific, gather feedback from, and interact with consumers.

\section{Materials and methods}

The purpose of this research was to examine the effects of the type of advertisement information presented in a testimonial versus testimonial and statistics, and the effect of age-appropriateness of models (age-appropriate versus age-inappropriate) on middle-aged women's perceptions of credibility, informativeness, believability, and likelihood to purchase a product presented in a Facebook sidebar advertisement for an anti aging beauty product. Because the primary analysis involved general linear modeling (e.g., analysis of variance), the variables in the study were examined for preliminary assumption testing, such as skewness and kurtosis. Variables that were found to violate the standard cutoffs for skewness and kurtosis were also examined using a test's nonparametric equivalent. The following research questions were addressed:

a. Q1: To what extent, if any, is there a difference in middle-aged women's perception of the credibility of an online sidebar advertisement for an anti aging product (rated on a 7-point Likert-type scale) based on the age of the model depicted in the advertisement (age-appropriate versus age-inappropriate) and the type of advertisement (testimonial only versus testimonial and statisti$\mathrm{cs})$ ?

b. Q2: To what extent, if any, is there a difference in middle-aged women's perception of the informativeness of an online sidebar advertisement for an anti aging product (rated on a 7-point Likert-type scale) based on the age of the model depicted in the advertisement (age-appropriate versus age-inappropriate) and the type of advertisement (testimonial only versus testimonial and statistics)?

c. Q3: To what extent, if any, is there a difference in middle-aged women's perception of the believability to purchase of an online sidebar advertisement for an anti aging product (rated on a 7-point Likert-type scale) based on the age of the model depicted in the advertisement (age-appropriate versus age-inappropriate) and the type of advertisement (testimonial only versus testimonial and statistics)?

d. Q4: To what extent, if any, is there a difference in middle-aged women's likelihood of purchasing an anti aging product depicted in an online sidebar advertisement (rated on a 7-point Likert-type scale) based on the age of the model depicted in the advertisement (age-appropriate versus age-inappropriate) and the type of advertisement (testimonial only versus testimonial and statistics)?

\section{Participants}

Frequencies and percentages for all categorical variables are displayed in Table 1. The majority of participants was White/Caucasian (95.4\%), reported a need for an anti-aging product (69.2\%), reported 
currently using an anti aging product (53.1\%), and reported that they had never made a purchase from social media advertising $(55.5 \%)$. In addition, approximately $25 \%$ of participants were randomly assigned to each of the advertisement conditions.

Table I Frequency and Percentages for Categorical Variables

\begin{tabular}{lll}
\hline Categorical variable & $\mathbf{n}$ & $\%$ \\
\hline Race & 370 & 95.4 \\
White/Caucasian & 3 & 0.8 \\
Black/African American & 2 & 0.5 \\
American Indian/Alaska Native & 2 & 0.5 \\
Asian & 1 & 0.3 \\
Native Hawaiian/Pacific Islander & 3 & 0.8 \\
Other & 7 & 1.8 \\
Two or more races & & \\
Need for anti aging product & 120 & 30.8 \\
No & 269 & 69.2 \\
Yes & & \\
Current use of anti aging product & 182 & 46.9 \\
No & 206 & 53.1 \\
Yes & & \\
Purchase from social media advertising & & \\
No & 216 & 55.5 \\
Yes & 173 & 44.5 \\
Advertisement condition & & \\
Age-appropriate and testimonial & 95 & 24.4 \\
Age-inappropriate and testimonial & 98 & 25.2 \\
Age-appropriate and testimonial with statistics & 98 & 25.2 \\
Age-inappropriate and testimonial with & 98 & 25.2 \\
statistics & & \\
\hline & & \\
\hline
\end{tabular}

Note Frequencies not summing to 389 reflect missing data

\section{Results}

Means and standard deviations for all continuous variables are displayed in Table 2. The mean age of participants was $42.40(\mathrm{SD}=7.34)$ with a minimum age of 30 and maximum age of 55 . On a scale from 1 (satisfied) to 7 (not satisfied), participants' average satisfaction with their current anti aging product was $2.67(\mathrm{SD}=1.69)$. Similarly, participants reported having a mostly neutral attitude toward social media advertising $(\mathrm{M}=4.14, \mathrm{SD}=1.69)$ on a scale from 1 (favorable) to 7 (unfavorable). On average, participants reported spending 56.39minutes a day on Facebook with a minimum of 1.50 and maximum of 650 . Because this variable was positively skewed, $\mathrm{Z}=39.63, \mathrm{p}<.001$, and leptokurtotic, $\mathrm{Z}=189.01, \mathrm{p}<.001$, the median is more descriptive of central tendency. The median number of Facebook minutes participants spent per day was 50minutes. For the dependent measures of credibility, informativeness, believability, and likelihood of purchasing the anti aging product the scaling was from 1([credible/ informative/believable/likely to buy]) to 7(not credible/informative/ believable/likely to buy]). Average scores for credibility were
5.15( $\mathrm{SD}=1.39)$, informativeness was $5.27(\mathrm{SD}=1.47)$, believability was $5.19(\mathrm{SD}=1.42)$, and likelihood of purchasing was $6.48(\mathrm{SD}=1.04)$ with minimum values of 1 and maximum values of 7 .

Table 2 Means and Standard Deviations for Continuous Variables

\begin{tabular}{|c|c|c|c|c|c|}
\hline Continuous variable & $\mathbf{N}$ & M & SD & Min & Max \\
\hline Age & 389 & 42.4 & 7.34 & 30 & 55 \\
\hline $\begin{array}{l}\text { Satisfaction with current } \\
\text { anti aging product }\end{array}$ & 291 & 2.67 & 1.69 & I & 7 \\
\hline $\begin{array}{l}\text { Attitude toward social } \\
\text { media advertising }\end{array}$ & 388 & 4.14 & 1.69 & I & 7 \\
\hline $\begin{array}{l}\text { Facebook use per day in } \\
\text { minutes }\end{array}$ & 387 & 56.39 & 51.58 & 1.5 & 650 \\
\hline Credibility & 389 & 5.15 & 1.39 & I & 7 \\
\hline Informativeness & 389 & 5.27 & 1.47 & I & 7 \\
\hline Believability & 389 & 5.19 & 1.42 & I & 7 \\
\hline $\begin{array}{l}\text { Likelihood of purchasing } \\
\text { anti aging product }\end{array}$ & 389 & 6.48 & 1.04 & I & 7 \\
\hline
\end{tabular}

Note Ns not equal to 389 reflect missing data

To test whether there was a relationship between need for and current use of anti aging products, a Pearson's chi-square test of independence was conducted (Table 3). As expected, there was a significant relationship between participants' reported use of an anti aging product and the need for an anti aging product, $\chi^{2}=150.36$, $\mathrm{p}<.001$. The proportion of women who use and need an anti aging product $(73.9 \%)$ was larger than the proportion of women who use but do not need an anti aging product $(6.7 \%)$.

Table 3 Frequencies and Percentages for Current Use of Anti aging Product by Need for Anti aging Product

\begin{tabular}{|c|c|c|c|c|c|c|c|}
\hline $\begin{array}{l}\text { Need for anti } \\
\text { aging product }\end{array}$ & & & & & & & \\
\hline \multirow[t]{2}{*}{$\begin{array}{l}\text { Demographic } \\
\text { variable }\end{array}$} & \multicolumn{2}{|l|}{ No } & \multicolumn{2}{|l|}{ Yes } & \multirow[t]{2}{*}{$\chi^{2}$} & \multirow[t]{2}{*}{$\mathbf{p}$} & \multirow[t]{2}{*}{$\phi$} \\
\hline & $n$ & $\%$ & $n$ & $\%$ & & & \\
\hline \multicolumn{5}{|l|}{$\begin{array}{l}\text { Current use } \\
\text { of anti aging } \\
\text { product }\end{array}$} & \multirow{3}{*}{150.36} & \multirow{3}{*}{$<.001$} & \multirow{3}{*}{0.623} \\
\hline No & 112 & $93.3^{\mathrm{a}}$ & 70 & $26.1^{b}$ & & & \\
\hline Yes & 8 & $6.7^{\mathrm{a}}$ & 198 & $73.9^{b}$ & & & \\
\hline
\end{tabular}

Note For each row category, pairs of column proportions with different superscripts differed significantly, $\mathrm{p}<.05$

$\Phi$ Phi coefficient reported

Pearson's product-moment correlations were calculated among all continuous demographic variables (Table 4). There was a significant negative correlation between Facebook use per day in minutes and age, $r=-.186, p<.001$. Older participants reported using Facebook less than did younger participants. The other correlations presented in Table 4 were not significant, ps $>.05$.

To assess the relationships between current use of an anti aging product by the continuous demographic variables, such as age, Facebook use, satisfaction with current anti aging product, and attitude toward social media, several independent sample's t 
tests were conducted (Table 5). There was a significant difference between participants who reported use of an anti aging product by age, $\mathrm{t}(386)=3.76, \mathrm{p}<.001, \mathrm{~d}=.383$. Participants who reported using an anti aging product $(\mathrm{M}=43.66, \mathrm{SD}=7.01)$ were significantly older than were participants who reported that they did not currently use an anti aging product $(\mathrm{M}=40.90, \mathrm{SD}=7.41)$. None of the other t-test statistics presented in Table 6 were significant, ps $>.05$.

Table 4 Pearson's Product-Moment Correlations among Continuous Demographic Variables

\begin{tabular}{llll}
\hline $\begin{array}{l}\text { Continuous } \\
\text { demographic } \\
\text { variable }\end{array}$ & Age & $\begin{array}{l}\text { Facebook use } \\
\text { per day in } \\
\text { minutes }\end{array}$ & $\begin{array}{l}\text { Satisfaction } \\
\text { with current } \\
\text { anti aging } \\
\text { product }\end{array}$ \\
\hline $\begin{array}{l}\text { Facebook use per } \\
\text { day in minutes }\end{array}$ & $-0.186^{* * * *}$ & & \\
$\begin{array}{l}\text { Satisfaction with } \\
\text { current anti aging } \\
\text { product }\end{array}$ & -0.11 & -0.072 & \\
$\begin{array}{l}\text { Attitude toward } \\
\text { social media } \\
\text { advertising }\end{array}$ & 0.077 & & \\
\hline
\end{tabular}

Note*** $\mathrm{p}<.001$

Several independent samples't tests were conducted to assess the differences between groups participants reported need for an anti aging product by continuous demographic variables (Table 6). There was a significant difference between participants who reported a need for an anti aging product by age, $\mathrm{t}(387)=3.15, \mathrm{p}=.002, \mathrm{~d}=.344$. Participants who reported a need for an anti aging product $(\mathrm{M}=43.17$,
$\mathrm{SD}=7.21)$ were significantly older than were participants who reported no need for an anti aging product $(\mathrm{M}=40.66, \mathrm{SD}=7.36)$. In addition, participants who reported a need for an anti aging product reported that they were significantly less satisfied with their anti aging product $(\mathrm{M}=2.89, \mathrm{SD}=1.72)$ than were participants who reported no need for an anti aging product $(\mathrm{M}=1.89, \mathrm{SD}=1.31), \mathrm{t}(289)=4.31$, $\mathrm{p}<.001, \mathrm{~d}=.661$. The other t-test statistics presented in Table 7 were nonsignificant, $\mathrm{ps}>.05$.

To assess whether or not participants made a purchase from social media advertising with continuous demographic variables, several independent sample's $t$ tests were conducted. As shown in Table 7, there was a significant relationship between whether participants had made a purchase from social media advertising and their attitude toward social media advertising, $\mathrm{t}(386)=8.08, \mathrm{p}<.001, \mathrm{~d}=.831$. Participants who had made a purchase from social media advertising reported more favorable attitudes toward social media advertising $(\mathrm{M}=3.42, \mathrm{SD}=1.55)$ than did participants who had not made a purchase from social media advertising $(\mathrm{M}=4.72, \mathrm{SD}=1.58)$. The other t-test statistics presented in Table 7 were not significant, $\mathrm{ps}>.05$.

\section{Relationships among dependent variables}

Several Pearson's product-moment correlations were calculated to assess the relationships among the dependent variables of believability, credibility, informativeness, and likelihood of purchasing the anti aging product presented in the advertisement. As shown in Table 8, all dependent variables were positively significantly correlated with each other, $\mathrm{ps}<.001$. The higher participants rated the advertisement on believability, credibility, informativeness, or likelihood to buy the anti aging product, the higher participants rated the advertisement on the other three measures.

Table 5 Means and Standard Deviations for Current Use of Anti aging Product by Demographic Variables

\begin{tabular}{lllllll}
\hline Variable & n & M & SD & t & P & d \\
\hline Age & & & & & & \\
No & 182 & 40.9 & 7.41 & & & \\
Yes & 206 & 43.66 & 7.01 & 3.76 & $<.001$ & 0.383 \\
Facebook Use Per Day in Minutes & & & & & & \\
No & 181 & 58.6 & 44.29 & & & \\
Yes & 205 & 54.13 & 57.23 & 0.85 & 0.396 & 0.088 \\
Satisfaction with Current Anti Aging Product $\Psi$ & & & & & & \\
No & 85 & 3.11 & 2.25 & & & \\
Yes & 205 & 2.49 & 1.36 & 2.36 & 0.02 & 0.344 \\
Attitude Toward Social Media Advertising & & & & & & \\
No & 181 & 4.25 & 1.74 & & & \\
Yes & 206 & 4.05 & 1.66 & & & \\
\hline
\end{tabular}

Note $\Psi$ Equal variances not assumed statistics reported

Table 6 Means and Standard Deviations for Need for Anti aging Product by Demographic Variables

\begin{tabular}{lllllll}
\hline Variable & n & M & SD & t & P & d \\
\hline Age & & & & & & \\
No & 120 & 40.66 & 7.36 & & & \\
Yes & 269 & 43.17 & 7.21 & 3.15 & 0.002 & 0.344 \\
Face book use per day in minutes & & & & & & \\
\hline
\end{tabular}


Table Continued..

\begin{tabular}{lllllll}
\hline Variable & $\mathbf{n}$ & $\mathbf{M}$ & SD & $\mathbf{t}$ & $\mathbf{p}$ & $\mathbf{d}$ \\
\hline No & 120 & 62.53 & 47.53 & & \multirow{2}{*}{0.117} & 0.177 \\
Yes & 267 & 53.64 & 53.16 & & & \\
Satisfaction with current anti aging product & & & & & & \\
No & 64 & 1.89 & 1.31 & & & \\
Yes & 227 & 2.89 & 1.72 & 4.31 & $<.001$ & 0.661 \\
Attitude toward social media advertising & & & & & & \\
No & 120 & 4.31 & 1.79 & & & \\
Yes & 268 & 4.07 & 1.65 & 1.28 & 0.202 & 0.14
\end{tabular}

Table 7 Means and Standard Deviations for Purchase from Social Media by Demographic Variables

\begin{tabular}{|c|c|c|c|c|c|c|}
\hline Variable & $\mathbf{n}$ & $\mathbf{M}$ & SD & $\mathbf{t}$ & $\mathbf{p}$ & d \\
\hline \multicolumn{7}{|l|}{ Age } \\
\hline No & 216 & 42.31 & 7.29 & \multirow{2}{*}{0.26} & \multirow{2}{*}{0.797} & \multirow{2}{*}{0.026} \\
\hline Yes & 173 & 42.5 & 7.43 & & & \\
\hline \multicolumn{7}{|c|}{ Face book use per day in minutes } \\
\hline No & 215 & 52.32 & 56.27 & \multirow{2}{*}{0.26} & \multirow{2}{*}{0.797} & \multirow{2}{*}{0.181} \\
\hline Yes & 172 & 61.48 & 44.7 & & & \\
\hline \multicolumn{7}{|c|}{ Satisfaction with current anti aging product } \\
\hline No & 158 & 2.71 & I.7I & \multirow{2}{*}{0.43} & \multirow{2}{*}{0.67} & \multirow{2}{*}{0.053} \\
\hline Yes & 133 & 2.62 & 1.66 & & & \\
\hline \multicolumn{7}{|c|}{ Attitude toward social media advertising } \\
\hline No & 216 & 4.72 & 1.58 & \multirow{2}{*}{8.08} & \multirow{2}{*}{$<.001$} & \multirow{2}{*}{0.831} \\
\hline Yes & 172 & 3.42 & 1.55 & & & \\
\hline
\end{tabular}

Table 8 Pearson's Product-Moment Correlations among Dependent Variables

\begin{tabular}{|c|c|c|c|}
\hline Continuous demographic variable & Believability & Credibility & In formativeness \\
\hline Credibility & $0.731^{* * *}$ & & \\
\hline In formativeness & $0.52 * * *$ & $0.56 * * *$ & \\
\hline Likelihood of purchasing anti aging product & $0.4 * * *$ & $0.429 * * *$ & $0.393 * * *$ \\
\hline
\end{tabular}

Note***p $<.001$

\section{Relationships between demographic and independent variables}

To ensure that all experimental groups were similar before being exposed to the manipulation, Pearson's chi-square tests of independence were conducted to assess whether there were significant associations between the advertisement condition and categorical demographic variables. As shown in Table 9, there were no significant differences found among the experimental conditions based upon participants' current use of an anti aging product, $\mathrm{p}>.05$.

As shown in Tables 10, there were no significant differences among experimental advertisement conditions based upon participants' reported need for an anti aging product, $\mathrm{p}>.05$.

Based upon the Pearson's chi-square test of independence, there was no association between the advertisement condition and whether participants had reported purchasing a product from social media advertising, $p>.05$ (Table 11).
Several ANOVAs were conducted to assess the bivariate relationships between advertisement condition and continuous demographic variables (Table 12a) (Table 12b). There were no significant differences found among the four experimental groups regarding age, satisfaction with current anti aging product, attitude toward social media advertising, and Facebook use per day in minutes, ps $>.05$.

\section{Relationships between demographic and dependent variables}

Several independent samples't tests were conducted on the dependent variables using need for anti aging product as the betweengroups variable (Table 13a) (Table 13b). There were no significant differences found in believability, credibility, informativeness, and likelihood of purchasing an anti aging product by whether or not participants reported a need for an anti aging product, $\mathrm{ps}>05$.

(Table 14a) (Table 14b) presents the means and standard deviations for the study's dependent variables by reported use of an anti aging 
product. The results of the independent sample's t tests suggest that informativeness, and likelihood of purchasing an anti aging product there is no significance between groups the believability, credibility, with current use of an anti aging product, ps>.05.

Table 9 Frequencies and Percentages for Current Use of Anti aging Product by Advertisement Condition

\begin{tabular}{|c|c|c|c|c|c|c|c|}
\hline \multicolumn{8}{|l|}{ Current use of anti aging product } \\
\hline \multirow[t]{2}{*}{ Demographic variable } & \multicolumn{2}{|l|}{ No } & \multicolumn{2}{|c|}{ Yes } & \multicolumn{3}{|c|}{ Cramer's } \\
\hline & $\mathbf{n}$ & $\%$ & $\mathbf{n}$ & $\%$ & $\chi^{2}$ & $\mathbf{p}$ & $\mathbf{V}$ \\
\hline \multicolumn{8}{|l|}{ Advertisement condition } \\
\hline Age-appropriate and testimonial & 49 & 26.9 & 45 & 21.8 & \multirow{4}{*}{3.35} & \multirow{4}{*}{0.341} & \multirow{4}{*}{0.093} \\
\hline Age-inappropriate and testimonial & $4 I$ & 22.5 & 57 & 27.7 & & & \\
\hline Age-appropriate and testimonial with statistics & 42 & 23.1 & 56 & 27.2 & & & \\
\hline Age-inappropriate and testimonial with statistics & 50 & 27.5 & 48 & 23.3 & & & \\
\hline
\end{tabular}

Table 10 Frequencies and Percentages for Need for Anti aging Product by Advertisement Condition

\begin{tabular}{|c|c|c|c|c|c|c|c|}
\hline \multicolumn{8}{|l|}{ Need for anti aging product } \\
\hline \multirow[t]{2}{*}{ Demographic variable } & \multicolumn{2}{|l|}{ No } & \multicolumn{2}{|c|}{ Yes } & \multicolumn{3}{|c|}{ Cramer's } \\
\hline & $\mathbf{n}$ & $\%$ & n & $\%$ & $\chi^{2}$ & $\mathbf{p}$ & $\mathbf{v}$ \\
\hline \multicolumn{8}{|l|}{ Advertisement condition } \\
\hline Age-appropriate and testimonial & 29 & 24.2 & 66 & 24.5 & \multirow{4}{*}{0.42} & \multirow{4}{*}{0.936} & \multirow{4}{*}{0.936} \\
\hline Age-inappropriate and testimonial & 31 & 25.8 & 67 & 24.9 & & & \\
\hline Age-appropriate and testimonial with statistics & 28 & 23.3 & 70 & 26 & & & \\
\hline Age-inappropriate and testimonial with statistics & 32 & 26.7 & 66 & 24.5 & & & \\
\hline
\end{tabular}

Table II Frequencies and Percentages for Purchase from Social media by Advertisement Condition

\begin{tabular}{|c|c|c|c|c|c|c|c|}
\hline \multicolumn{8}{|l|}{ Purchase from social media advertising } \\
\hline \multirow[t]{2}{*}{ Demographic variable } & \multicolumn{2}{|l|}{ No } & \multicolumn{2}{|c|}{ Yes } & \multicolumn{3}{|c|}{ Cramer's } \\
\hline & $\mathbf{n}$ & $\%$ & $\mathbf{n}$ & $\%$ & $\chi^{2}$ & $\mathbf{p}$ & $\mathbf{v}$ \\
\hline \multicolumn{8}{|l|}{ Advertisement condition } \\
\hline Age-appropriate and testimonial & 56 & 25.9 & 39 & 22.5 & \multirow{4}{*}{4.26} & \multirow{4}{*}{0.235} & \multirow{4}{*}{0.105} \\
\hline Age-inappropriate and testimonial & 49 & 22.7 & 49 & 28.3 & & & \\
\hline Age-appropriate and testimonial with statistics & 50 & 23.1 & 48 & 27.7 & & & \\
\hline Age-inappropriate and testimonial with statistics & 61 & 28.2 & 37 & 21.4 & & & \\
\hline
\end{tabular}

Table I 2a Means and Standard Deviations for Continuous Demographic Variables by Advertisement Condition

\begin{tabular}{|c|c|c|c|c|c|c|}
\hline Variable by condition & $\mathbf{n}$ & $\mathbf{M}$ & SD & $\mathbf{F}$ & $\mathbf{p}$ & $\eta^{2}$ \\
\hline Age Age-appropriate and testimonial & 95 & 43.42 & 7.49 & \multirow{4}{*}{0.98} & \multirow{4}{*}{0.401} & \multirow{4}{*}{0.008} \\
\hline Age-inappropriate and testimonial & 98 & 41.64 & 7.17 & & & \\
\hline Age-appropriate and testimonial with statistics & 98 & 42.21 & 7.27 & & & \\
\hline Age-inappropriate and testimonial with statistics & 98 & 42.34 & 7.44 & & & \\
\hline \multicolumn{7}{|l|}{ Satisfaction with current anti aging product } \\
\hline Age-appropriate and testimonial & 62 & 2.73 & 1.72 & \multirow{5}{*}{0.33} & \multirow{5}{*}{0.806} & \multirow{4}{*}{0.003} \\
\hline Age-inappropriate and testimonial & 77 & 2.51 & 1.6 & & & \\
\hline Age-appropriate and testimonial with statistics & 81 & 2.73 & $1.6 \mathrm{I}$ & & & \\
\hline Age-inappropriate and testimonial with statistics & 7I & 2.73 & 1.85 & & & \\
\hline Attitude toward social media advertising & & & & & & \\
\hline
\end{tabular}


Table Continued.

\begin{tabular}{|c|c|c|c|c|c|c|}
\hline Variable by condition & $\mathbf{n}$ & $\mathbf{M}$ & SD & $\mathbf{F}$ & $\mathbf{p}$ & $\eta^{2}$ \\
\hline Age-appropriate and testimonial & 95 & 4.21 & 1.7 & \multirow{4}{*}{0.31} & \multirow{4}{*}{0.821} & \multirow{4}{*}{0.002} \\
\hline Age-inappropriate and testimonial & 98 & 4.21 & 1.63 & & & \\
\hline Age-appropriate and testimonial with statistics & 97 & 4.01 & 1.7 & & & \\
\hline Age-inappropriate and testimonial with statistics & 98 & 4.14 & 1.76 & & & \\
\hline
\end{tabular}

Table I 2b Means and Standard Deviations for Continuous Demographic Variables by Advertisement Condition

\begin{tabular}{|c|c|c|c|c|c|c|}
\hline Variable by condition & $\mathbf{n}$ & $\mathbf{M}$ & SD & $\mathbf{F}$ & $\mathbf{p}$ & $\eta^{2}$ \\
\hline \multicolumn{7}{|l|}{ Face book use per day in minutes } \\
\hline Age-appropriate and testimonial & 95 & 54.07 & 40.88 & \multirow{4}{*}{0.11} & \multirow{4}{*}{0.957} & \multirow{4}{*}{0.001} \\
\hline Age-inappropriate and testimonial & 97 & 56.95 & 45.27 & & & \\
\hline Age-appropriate and testimonial with statistics & 98 & 58.16 & 69.16 & & & \\
\hline Age-inappropriate and testimonial with statistics & 97 & 56.32 & 46.6 & & & \\
\hline
\end{tabular}

Table I 3a Means and Standard Deviations for Need for Anti aging Product by Dependent Variables

\begin{tabular}{lllllll}
\hline Variable & $\mathbf{n}$ & $\mathbf{M}$ & SD & $\mathbf{t}$ & $\mathbf{p}$ & $\mathbf{d}$ \\
\hline Believability & & & & & & \\
No & 120 & 5.28 & 1.44 & & & \\
Yes & 269 & 5.15 & $1.4 \mathrm{I}$ & & & \\
Credibility & & & & & & \\
No & 120 & 5.23 & 1.37 & & & \\
Yes & 269 & 5.12 & 1.39 & & & 0.604 \\
\hline
\end{tabular}

Table I3b Means and Standard Deviations for Need for Anti aging Product by Dependent Variables

\begin{tabular}{|c|c|c|c|c|c|c|}
\hline Variable & $\mathbf{n}$ & $\mathbf{M}$ & SD & $\mathbf{t}$ & $\mathbf{p}$ & d \\
\hline \multicolumn{7}{|c|}{ In formativeness } \\
\hline No & 120 & 5.26 & 1.53 & \multirow{2}{*}{0.1} & \multirow{2}{*}{0.917} & \multirow{2}{*}{0.011} \\
\hline Yes & 269 & 5.28 & 1.45 & & & \\
\hline \multicolumn{7}{|c|}{ Likelihood of purchasing anti aging product } \\
\hline No & 120 & 6.58 & 0.96 & \multirow{2}{*}{1.27} & \multirow{2}{*}{0.204} & \multirow{2}{*}{0.143} \\
\hline Yes & 269 & 6.44 & 1.07 & & & \\
\hline
\end{tabular}

Table I4a Means and Standard Deviations for Current Use of Anti aging Product by Dependent Variables

\begin{tabular}{|c|c|c|c|c|c|c|}
\hline Variable & $\mathbf{n}$ & $\mathbf{M}$ & SD & $\mathbf{t}$ & $\mathbf{p}$ & d \\
\hline \multicolumn{7}{|c|}{ Believability } \\
\hline No & 182 & 5.21 & I.47 & 0.37 & 0.708 & 0.038 \\
\hline Yes & 206 & 5.16 & $\mathrm{I} .37$ & & & \\
\hline \multicolumn{7}{|l|}{ Credibility } \\
\hline No & 182 & 5.21 & 1.42 & 0.83 & 0.407 & 0.084 \\
\hline Yes & 206 & 5.1 & 1.35 & & & \\
\hline
\end{tabular}


Table I4b Means and Standard Deviations for Current Use of Anti aging Product by Dependent Variables

\begin{tabular}{|c|c|c|c|c|c|c|}
\hline Variable & $\mathbf{n}$ & $\mathbf{M}$ & SD & $\mathbf{t}$ & $\mathbf{p}$ & d \\
\hline \multicolumn{7}{|c|}{ In formativeness } \\
\hline No & 182 & 5.3 & 1.5 & \multirow{2}{*}{0.43} & \multirow{2}{*}{0.668} & \multirow{2}{*}{0.044} \\
\hline Yes & 206 & 5.24 & 1.45 & & & \\
\hline \multicolumn{7}{|c|}{ Likelihood of purchasing anti aging product } \\
\hline No & 182 & 6.53 & 1.05 & \multirow{2}{*}{0.81} & \multirow{2}{*}{0.418} & \multirow{2}{*}{0.083} \\
\hline Yes & 206 & 6.44 & 1.03 & & & \\
\hline
\end{tabular}

Several independent sample's t tests were conducted to assess the relationships between believability, credibility, informativeness, and likelihood of purchasing an anti aging product with whether or not participants had made a purchase from social media advertising (Table 15). There was a significant between groups found between believability and whether or not participants had made a purchase from social media advertising, $\mathrm{t}(387)=2.17, \mathrm{p}=.030, \mathrm{~d}=.221$. Participants who had previously made a purchase from social media advertising $(\mathrm{M}=5.01, \mathrm{SD}=1.48)$ reported that the advertisement ad was more believable than did participants who had reported that they had never made a purchase from social media advertising $(M=5.32, S D=1.35)$. In addition, there was a significant relationship found between purchase from social media advertising and credibility, $\mathrm{t}(387)=2.27, \mathrm{p}=.024$, $\mathrm{d}=.232$. Participants who had previously made a purchase from social media advertising $(\mathrm{M}=5.13, \mathrm{SD}=1.47)$ reported that the advertisement ad was more credible than did participants who had reported that they had never made a purchase from social media advertising $(\mathrm{M}=5.30$, $\mathrm{SD}=1.38$ ). There were no significant between groups found between purchase from social media advertising with informativeness or likelihood of purchasing the anti aging product, $\mathrm{ps}>.05$.

To examine whether significant bivariate relationships existed between the dependent measures and the continuous demographic variables, several Pearson's product-moment correlation coefficients were calculated (Table 16). Age was significantly associated with credibility, $\mathrm{r}=-.126, \mathrm{p}=.013$. Older participants were more likely to report lower advertisement credibility ratings, suggesting that older participants rated the advertisement as more credible than did younger participants. In addition, participants' attitude toward social media advertising was positively correlated with believability, $\mathrm{r}=.225, \mathrm{p}<.001$, credibility, $\mathrm{r}=.284, \mathrm{p}<.001$, informativeness, $\mathrm{r}=.204$, $\mathrm{p}<.001$, and likelihood of purchasing the anti aging product, $\mathrm{r}=.190$, $\mathrm{p}<.001$. The more favorable participants' attitude toward social media advertising, the more likely they were to view the advertisement as believable, credible, and informative. Participants with more favorable attitudes toward social media advertising were also more likely to report higher purchase intentions of the anti aging product. None of the other correlations reported in Table 16 were significant, ps $>.05$.

Table 15 Means and Standard Deviations for Purchase from Social Media by Dependent Variables

\begin{tabular}{|c|c|c|c|c|c|c|}
\hline Variable & $\mathbf{n}$ & M & SD & $\mathbf{t}$ & $\mathbf{p}$ & d \\
\hline \multicolumn{7}{|c|}{ Believability } \\
\hline No & 216 & 5.32 & 1.35 & \multirow{2}{*}{2.17} & \multirow{2}{*}{0.03} & \multirow{2}{*}{0.221} \\
\hline Yes & 173 & 5.01 & 1.48 & & & \\
\hline \multicolumn{7}{|c|}{ Credibility } \\
\hline No & 216 & 5.3 & 1.38 & \multirow{2}{*}{2.27} & \multirow{2}{*}{0.024} & \multirow{2}{*}{0.232} \\
\hline Yes & 173 & 4.98 & 1.38 & & & \\
\hline \multicolumn{7}{|c|}{ In formativeness } \\
\hline No & 216 & 5.38 & 1.46 & \multirow{2}{*}{1.65} & \multirow{2}{*}{0.1} & \multirow{2}{*}{0.168} \\
\hline Yes & 173 & 5.13 & 1.47 & & & \\
\hline \multicolumn{7}{|c|}{ Likelihood of purchasing anti aging product } \\
\hline No & 216 & 6.51 & 1.03 & \multirow{2}{*}{0.65} & \multirow{2}{*}{0.516} & \multirow{2}{*}{0.066} \\
\hline Yes & 173 & 6.45 & 1.04 & & & \\
\hline
\end{tabular}

Table 16 Pearson's Product-Moment Correlations for Continuous Demographic Variables with Dependent Variables

\begin{tabular}{lllll}
\hline Variable & Age & $\begin{array}{l}\text { Face book use per } \\
\text { day in minutes }\end{array}$ & $\begin{array}{l}\text { Satisfaction with current } \\
\text { anti aging product }\end{array}$ & $\begin{array}{l}\text { Attitude toward social media } \\
\text { advertising }\end{array}$ \\
\hline Believability & -0.068 & 0.005 & 0.047 & 0.225 \\
Credibility & $-0.126^{*}$ & 0.009 & -0.003 & $0.284^{* * *}$ \\
$\begin{array}{l}\text { In formativeness } \\
\text { Likelihood of purchasing } \\
\text { anti aging product }\end{array}$ & -0.044 & 0.009 & -0.022 & $0.204^{* * *}$ \\
\hline
\end{tabular}

Note*p<.05**p<.01***p<.001 


\section{Primary analysis}

To answer the primary research questions, a multiple analysis of covariance (MANCOVA) test was conducted on the four dependent variables of credibility, informativeness, believability, and likelihood to purchase the anti aging product. The independent variable, therefore, was advertisement condition. Because the preliminary analyses determined that age, attitude toward social media advertising, and purchase from social media were significantly related to one or more of the dependent measures, they were included in the analysis as covariates. The MANCOVA met the appropriate cutoffs for the test of equality of covariance matrices, Box's M=52.74, F (30, 404940.26) $=1.73, \mathrm{p}=.008$. Although Box's M test was significant, this test is sensitive to sample size, which is why recommended cutoffs for violations are suggested at $\mathrm{p}<.001$ for large samples (Tabachnick $\&$ Fidell, 2007). According to the Levene's test of equality of error variances, error variances were similar across all experimental groups, $\mathrm{ps}>.05$. Table 18 presents the means and standard deviations for the dependent measures by advertisement condition, as well as the inferential results of the MANCOVA. The multivariate effect of the MANCOVA was significant, $F(12,1000.385)=2.27, \mathrm{p}=.008$, $\eta_{\mathrm{p}}^{2}=.023$. The significant multivariate effect suggests that when Table 17 Means and Standard Deviations for Dependent Variables by Advertisement Condition

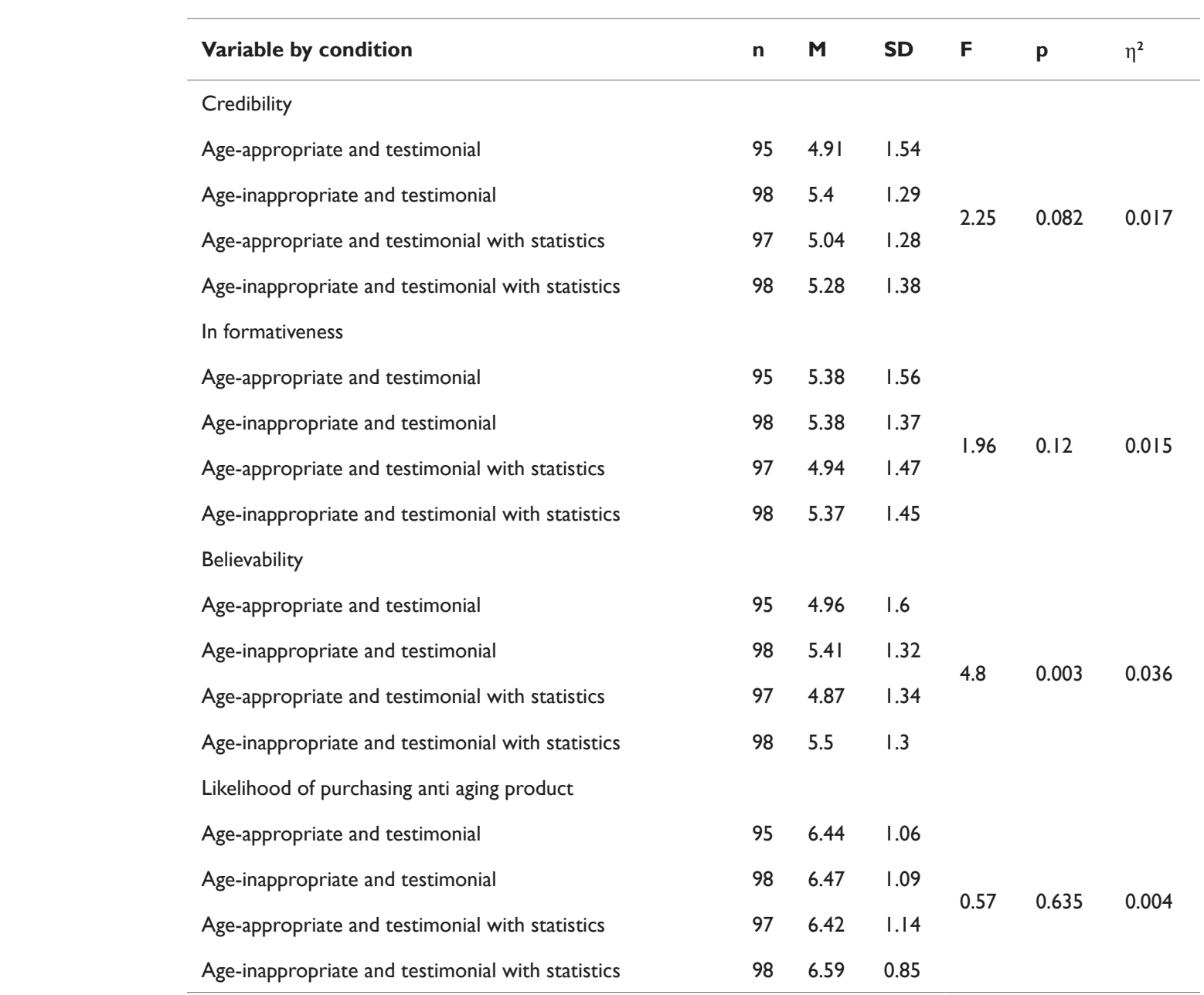

Note Age, attitude toward social media advertising, and purchase from social media advertising entered into the MANOVA model as covariates. Multivariate

$F(I 2,1000.385)=2.27, p=.008, \eta^{2} p=.023$. Means with different superscripts differ significantly, $p<.05$ controlling for age, attitude toward social media advertising, and purchase from social media advertising, there was a significant effect of advertisement condition on the linear combination of credibility, informativeness, believability, and likelihood of purchasing anti aging product. Based upon the significant multivariate effect, the univariate effects were further explored to answer the specific research questions and hypotheses.

Research Question 1 asked to what extent, if any, is there a difference in middle-aged women's perception of the credibility of an online sidebar advertisement for an anti aging product (rated on a 7-point Likert-type scale) based on the age of the model depicted in the advertisement (age-appropriate versus age-inappropriate) and the type of advertisement (testimonial only versus testimonial and statistics). Based upon the univariate analyses from the MANCOVA, advertisement condition did not significantly affect participants' credibility ratings when controlling for age, attitude toward social media advertising, and purchase from social media advertising, $\mathrm{F}(3,381)=2.25, \quad \mathrm{p}=.082, \quad \eta_{\mathrm{p}}^{2}=.017$. Participants across the four experimental conditions, therefore, reported similar credibility ratings (Table 17). 
Research Question 2 asked to what extent, if any, is there a difference in middle-aged women's perception of the informativeness of an online sidebar advertisement for an anti aging product (rated on a 7-point Likert-type scale) based on the age of the model depicted in the advertisement (age-appropriate versus age-inappropriate) and the type of advertisement (testimonial only versus testimonial and statistics). According to the univariate analysis from the MANCOVA, advertisement condition did not significantly affect informativeness ratings, $\mathrm{F}(3,381)=1.96, \mathrm{p}=.120, \eta_{\mathrm{p}}^{2}=.015$. Across the four experimental conditions, participants reported similar informativeness ratings of the ad (Table 18).

Research Question 3 asked to what extent, if any, is there a difference in middle-aged women's perception of the believability to purchase of an online sidebar advertisement for an anti aging product (rated on a 7-point Likert-type scale) based on the age of the model depicted in the advertisement (age-appropriate versus ageinappropriate) and the type of advertisement (testimonial only versus testimonial and statistics). Results from the univariate analyses from the MANCOVA revealed that when controlling for age, attitude toward social media advertising, and purchase from social media advertising, there was a significant main effect of advertisement condition on believability ratings (Table 18$), \mathrm{F}(3,381)=4.80, \mathrm{p}=.003, \eta^{2}=.036$. To further explore this main effect, post hoc analyses were conducted using a Bonferroni adjustment for multiple comparisons. Participants exposed to the age-inappropriate and testimonial advertisement with statistics $(M=5.50, \mathrm{SD}=1.30)$ reported lower believability ratings than did participants exposed to the age-appropriate and testimonial with statistics advertisement $(\mathrm{M}=4.87, \mathrm{SD}=1.34)$ and participants exposed to age-appropriate and testimonial advertisement $(\mathrm{M}=4.96, \mathrm{SD}=1.60)$, ps $<.05$. There were no significant differences between the other advertisement conditions regarding believability ratings, ps $>05$.

Research Question 4 asked to what extent, if any, is there a difference in middle-aged women's likelihood of purchasing an anti aging product depicted in an online sidebar advertisement (rated on a 7-point Likert-type scale) based on the age of the model depicted in the advertisement (age-appropriate versus age-inappropriate) and the type of advertisement (testimonial only versus testimonial and statistics). Based upon the univariate analyses from the MANCOVA, advertisement condition did not significantly affect participants likelihood of purchasing the anti aging product when controlling for age, attitude toward social media advertising, and purchase from social media advertising, $\mathrm{F}<1$. Participants across the four experimental conditions, therefore, reported similar purchase intention ratings (Table 18)

\section{Results and discussion}

Based upon the results from the preliminary and primary analyses, only the null hypothesis for Research Question 3 was rejected. There was a significant difference found in middle-aged women's perception of the believability to purchase an advertisement depicted in an online sidebar advertisement for an anti aging product. This difference, furthermore, was based upon the age of the model depicted in the advertisement (age-appropriate versus age-inappropriate) and the type of advertisement (testimonial only versus testimonial and statistics). The null hypotheses for Research Questions 1-2 and 4 were retained; there were no significant differences found in middleaged women's perception of the credibility, informativeness, or likelihood of purchasing the anti aging product depicted in an online sidebar advertisement based upon the age of the model depicted in the advertisement or the type of advertisement.

These findings correlated with previous research about UGT, whereas consumers will connect with an advertisement when the content in an advertisement fulfills a need such as believability or relatability to the product Katz et al. ${ }^{14}$

\section{Summary}

Overall, when controlling for age, attitude toward social media advertising, and purchase from social media advertising, there was a significant effect of advertisement condition on believability ratings. Middle-aged, female Facebook users exposed to the age-inappropriate and testimonial advertisement with statistics reported lower believability ratings than did those exposed to the age-appropriate and testimonial with statistics advertisement and those exposed to ageappropriate and testimonial advertisement. There were no differences found in women's perception of the credibility, informativeness, or likelihood of purchasing the anti aging product depicted in the online sidebar advertisement based upon the advertisement model or type.

Although only one alternative hypothesis was retained out of the four hypotheses in the study, there were several significant findings that were not central to the study's hypotheses worth mentioning with regard to age, anti aging product use, Facebook use, attitude toward social media advertising, and purchase from social media advertising. Specifically, older women reported using Facebook less than younger women. In addition, older women were more likely to report using an anti aging product and report needing to use an anti aging product than younger women. Women who reported a need for an anti aging product were less satisfied with their current anti aging product than were women who reported using but not needing an anti aging product.

Regarding social media attitudes and previous purchasing behavior, women who had reported making a purchase from social media advertising reported more favorable attitudes toward social media advertising than did women who had not made a purchase from social media advertising. Women who had previously made a purchase from social media advertising also reported that the advertisement ad was more believable and credible than did participants who had reported that they had never made a purchase from social media advertising. Finally, women's attitude toward social media advertising was positively correlated with believability, credibility, informativeness, and likelihood of purchasing the anti aging product. Taken together, these results suggest that previous purchase behavior from social media advertising, age, and attitudes toward social media advertising are related to how middle-age women perceive sidebar advertisements for anti aging products. In addition, the content of the advertisement has relevance for how believable middle-aged women perceive anti aging products depicted in an online sidebar advertisement.

\section{Results}

The results show that there were no differences in middle-aged women's perception of credibility of an online sidebar advertisement for an anti aging product. There were no differences in middleaged women's perception of informativeness of an online sidebar advertisement for an anti aging product. There was a difference in middle-aged women's perception of believability of an online sidebar advertisement for anti aging product, such that (a) women who saw the age-appropriate and testimonial advertisement rated the advertisement as more believable than did women who viewed the age-inappropriate 
and testimonial with statistics advertisement and (b) women who saw the age-appropriate and testimonial with statistics rated the advertisement as more believable than did the women who viewed the age-inappropriate and testimonial with statistics advertisement. There were no differences in middle-aged women's perception of likelihood to purchase an anti aging product depicted in an online sidebar advertisement for an anti aging product.

The findings of the study can be considered with respect to past empirical findings. The main purpose of this section of the chapter is to triangulate the study findings with previous findings. In addition, any theoretical divergences between the current study and past studies also are noted. This section of the chapter has been structured to reflect the findings' implications for certain themes. The first subsection examines the first, second, and fourth research questions implications for trust-building. The second sub-section examines the third research question's implications for age denialism. The third sub-section addresses the research questions' collective implications for brand preferences.

building and information gathering: The first research question of the study was as follows: To what extent, if any, is there a difference in middle-aged women's perception of the credibility of an online sidebar advertisement for an anti aging product (rated on a 7-point Likert-type scale) based on the age of the model depicted in the advertisement (age-appropriate versus age-inappropriate) and the type of advertisement (testimonial only versus testimonial and statistics)? In answer to the first research question, it was found that there were no differences in middle-aged women's perception of credibility of an online sidebar advertisement for an anti aging product. The second research question focused on informativeness rather than credibility, while the fourth research question focused on purchasing likelihood as the independent variable. The findings of the first, second, and fourth research questions were at odds with the findings reported by Grieve. In Grieve's study, trust-building was found to emerge from a process of information gathering conducted on social media. This trust-building was found, in turn, to lead to higher purchase likelihood. Thus, in Grieve et al.'s sequential model, a particularly informative advertisement (such as an advertisement utilizing a combination of personal testimony and statistics, which was one of the experimental conditions of the current study) would be associated with a higher degree of perceived informativeness and higher purchase likelihood. However, in the current study, this finding was not obtained. Had this finding been replicated, there would have been a stronger correlation between informativeness and purchase intention than was actually measured in the study.

\section{Age denialism}

The findings for the third research question of the study appeared to be at odds with the empirical findings of Palmore (2007), insofar as the findings for the third research question supported the absence of age denialism in the sample whereas Palmore's findings supported the claim that one of the bases of the anti-aging cosmetics niche was of age denialism. However, if anti-aging commercials truly prompted or tapped into age denialism as experienced by middle-class or elderly consumers, then it is unlikely that such consumers would rate a commercial with a young model as lacking believability (which was the finding of the third research question of the study). It seems that individuals committed to age denialism would lack either the ability or the desire to judge the believability of an anti-aging commercial solely on the basis of the youth of the model. The fact that participants in the current study were indeed able to judge the anti-aging commercial to be less believable because of its association with a younger model undercuts Palmore's claim about the age denialism of consumers of anti-aging products.

\section{Brand preferences}

The findings of the study were closely aligned with ${ }^{27}$ findings. Murray found that consumers have very strong preferences for their own brands. Thus, even if consumers are purchasers of certain classes of products (such as cosmetics products), they are likely to treat different brands of cosmetics with different levels of loyalty. In the study, it was found that participants had a very low mean likelihood of purchasing the advertised product, despite the fact that most participants were avid users of anti-aging products and indicated that they felt the need to use such products. This finding is highly compatible with Murray's finding that loyalty to a certain class of products (which could be a reflection of the uses and gratifications associated with this product class) is not necessarily reflected in loyalty to several brands within this product class.

\section{Gaps in the empirical research base}

Although the findings of the study can be understood in relation to some past empirical findings, such as the findings described above, the subject matter of the study appeared to be unique in the context of the existing research base. As such, the results of the study are not directly comparable to other studies, none of which utilized the kind of approaches and models utilized in the current study. The results of the current study can therefore best be understood in the context of generating new information about the subject matter. ${ }^{28,29}$

\section{Conclusion}

The main theoretical framework chosen for the study was uses and gratifications theory (UGT) focused on the media channel Facebook for the social and psychological origins of (2) needs, which generate (3) expectations of (4) the mass media or other sources, which lead to (5) differential patterns of media exposure (or engagement in other activities), resulting in (6) need gratifications and (7) other consequences. (p. 20)

Since all the participants were Facebook consumers, participants responded to two specific aspects of Facebook content, namely (a) the age-appropriateness of a model and (b) the type of advertisement. Based on the theoretical logic of UGT, and on the experimental conditions of the study, the following questions were asked:

a. Why was variation in model age and type of advertisement not associated with variation in perceived credibility of an advertisement?

b. Why was variation in model age and type of advertisement not associated with variation in perceived informativeness of an advertisement?

c. Why was variation in model age and type of advertisement associated with variation in perceived believability of an advertisement?

d. Why was variation in model age and type of advertisement not associated with variation in perceived likelihood of purchasing a product depicted in an advertisement?

Participants had substantial uses for, and gratifications related to, 
the use of an anti-aging product. The results for the first, second, and fourth research questions is that the chosen experimental conditions were simply not relevant to the uses and gratifications experienced by the participants. The logic of this interpretation can be carried over to the results of the third research question. The fact that participants found the age-inappropriate model to be less believable could suggest that the participants, who were middle-aged, believed that the inordinate youth of the model made the product less relevant to a useful audience.

The results showed that it is possible that, in terms of credibility, informativeness, and purchase intention, model age and advertisement type were irrelevant to the uses and gratifications of the participants, possible because they were not given enough material to judge or because, in one way or another, the experimental conditions were irrelevant to uses and gratifications. In terms of believability, it is possible that the age-inappropriateness of the model was coded in terms of the product being less useful to what was, after all, a sample of middle-aged women.

One of the main statistics in support of the UGT interpretation offered above is that the likelihood of purchasing the product had a mean value of 6.5 , with a score of 7 indicated the maximum possible unlikelihood of not buying the product. Overwhelmingly, participants were not interested in the product being advertised, which, in terms of UGT, means that participants did not associate either uses or gratifications with the product. The statistical analysis found significant and positive cross-correlations between credibility, believability, informativeness, and likelihood of buying the product. This empirical finding was closely aligned with UGT, because, in UGT, consumption is the result of positive orientation towards a product or service. Thus, someone inclined to find an advertisement for a product to be informative, credible, and believable also would also be inclined to purchase that product. Because most of the participants in the current study were unlikely to purchase the product, they also tended to find the advertisement lacking in credibility, believability, and informativeness, and variations in the model age and advertisement type were not able to outweigh the absence of perceived uses and gratifications.

Statistically, the main finding of the study was that the experimental conditions exerted no predictive power over perceived credibility, informativeness, and intention of purchasing, and little predictive power over believability. In terms of empirical studies, the current study's findings were found to either contradict or fail to confirm some previous studies' findings related to the characteristics of antiaging product users and to information-based theories of purchase likelihood and positive product assessment. However, because of the novelty of the study, its results are not directly comparable to most of the existing research findings; the results are better understood as contributing to the development of new knowledge about the assessment of advertisements delivered through the Facebook platform.

In theoretical terms, the findings can be interpreted in alignment with UGT, insofar as participants were unlikely to consume the product and therefore unlikely to ascribe uses and gratifications to it based on the factors of believability, credibility, and informativeness. However, the fact that participants' assessments of the advertisements' believability, credibility, informativeness, and likelihood of being purchased were not related to either (a)participants' use of anti-aging products and (b) participants' perceived need for anti-aging products was not as easy to reconcile with UGT. Recommendations for future scholarship included (a) adding more experimental conditions, (b) gathering some qualitative data, and (c) measuring the impact of social media friendships on the assessment of products or services advertised through Facebook.

\section{Acknowledgements}

None.

\section{Conflict of interest}

Author declares there is no conflict of interest in publishing the article.

\section{References}

1. Benbow Buitenhuis A. A feminine Double-bind? Towards understanding the commercialization of beauty through examining anti-ageing culture. Social Alternatives. 2014;33(2):43-49.

2. Flores W, Chen JCV, Ross WH. The effect of variations in banner ad, type of product, website context, and language of advertising on Internet users' attitudes. Computers in Human Behavior. 2014;31:37-47.

3. Van Hellemont C, Van den Bulck H. Impacts of advertisements that are unfriendly to women and men. International $J$ Advertising. 2012;31(3):623-656.

4. The Dove campaign for real beauty. 2015.

5. Iyer R, Reisenwitz T. Understanding cognitive age: The boomers' perspective. Marketing Management J. 2010;20(2):28-41.

6. Kozar JM. Women's responses to fashion media images: A study of female consumers aged 30-59. International J Consumer Studies. 2010;34(3):272-278.

7. O Neil A. A call for truth in the fashion pages: What global trend in advertising regulation means for U.S. beauty and fashion advertisers. Indiana J Global Legal Studies. 2014;21(2):619-641.

8. Williams KC, Page RA, Petrosky AR, et al. Multi-generational marketing: Descriptions, characteristics, lifestyles, and attitudes. J Applied Business \& Economics. 2010;11(2):21-36.

9. Brown A, Knight T. Shifts in media images of women appearance and social status from 1960 to 2010: A content analysis of beauty advertisements in two Australian magazines. J Aging Studies. 2015;35(1):74-76.

10. Kozar JM. Effects of model age on adult female consumers' purchase intentions and attitudes for an age-specific product, clothing. International J Marketing Studies. 2012;4(2):22-29.

11. Rossiter JR, Percy L. Observations: How the roles of advertising merely appear to have changed. International $J$ Advertising. 2013;32(3):391-398.

12. Williams KC, Page RA. Marketing to the generations. J Behavioral Studies in Business. 2011;3:1-17.

13. Grant R. Uses and gratification theory. In: West RL, Turner LH, editors. Introducing communication theory: Analysis and application. 6th ed. Boston, MA: McGraw-Hill; 2010. p. 392-409.

14. Katz E, Blumler JG, Gurevitch M. Uses and gratifications research. Public Opinion Quarterly. 1973;37(4):509-523.

15. Sobh R. Mirror, mirror: Youth quest for middle-aged women. Latin American Advances in Consumer Research. 2008;2:120-125.

16. Kim YJ, Han J. Why smartphone advertising attracts customers: A model of web advertising, flow, and personalization. Computers in Human Behavior. 2014;33(1):256-269. 
17. Tsang MM, Ho SC, Liang TP. Consumer attitudes toward mobile advertising: An empirical study. International J Electronic Commerce. 2004;8(3):65-78

18. Yu SY, Park EA, Sung M. Cosmetics advertisements in women's magazines: A cross-cultural analysis of China and Korea. Social Behavior and Personality: An International J. 2015;43(4):685-704.

19. Clements K. Former Vogue editor: The truth about size zero. 2013.

20. Yu UJ, Damhorst ML, Russell DW. The impact of body image on consumers' perceptions of idealized advertising images and brand attitudes. Family \& Consumer Sciences Research J. 2011;40(1):58-73.

21. Meng J, Gonzenbach WJ, Pan PL. Third-person perception of cosmeceutical product advertising: The moderating role of body esteem. $J M e-$ dical Marketing. 2014;14(2-3):163-171.

22. Phillips BJ, McQuarrie EF. Narrative and persuasion in fashion advertising. J Consumer Research. 2010;37(3):368-392.

23. Dinner IM, Van Heerde HJ, Neslin S. Driving online and offline sales: The cross-channel effects of traditional, online display, and paid search advertising. J Marketing Research. 2014;51(5):527-545.
24. Carter B. Why Facebook ads are the biggest marketing opportunity ever. 2014.

25. Herzog H. Professor quiz: A gratification study. In: Lazarsfeld PF, editor. Radio and the printed page: An introduction to the study of radio and its role in the communication of ideas. Duell, Sloan, and Pearce, New York; 1940. p. 354.

26. Herzog H. What do we really know about daytime serial listeners? In: Lazarsfeld PF, Stanton FN, editors. Radio research 1942-1943. Duell, Sloan, and Pearce, New York, USA; 1944. p. 3-33.

27. Murray KB. Loyalty by design: Understanding consumers' reluctance to shop when buying online. Advances in Consumer Research. 2002;29(1):8-10.

28. Lazarsfeld PF. Radio and the printed page. Duell, Sloan, and Pierce, New York, USA; 1940. p. 386.

29. Littlejohn YS, Guba E. Encyclopedia of communication theory. Thousand Oaks: Sage publications; 2009. p. 1174. 